\title{
Implications of recurrent SARS-CoV-2 outbreaks for IBD management
}

\author{
Jonathan P Segal (D) , ${ }^{1}$ Alan C Moss (D) ${ }^{2}$
}

${ }^{1}$ Gastroenterology and Hepatology, St Mary's Hospital, London, UK

${ }^{2}$ Gastroenterology, BIDMC/ Harvard Medical School, Boston, Massachusetts, USA

\section{Correspondence to} Dr Jonathan P Segal, Gastroenterology and Hepatology, St Mary's Hospital, London W2 1NY, UK: jonathansegal1@nhs.net

Received 4 May 2020 Revised 9 June 2020 Accepted 14 June 2020 Published Online First 24 June 2020

\section{Check for updates}

(C) Author(s) (or their employer(s)) 2021. No commercial re-use. See rights and permissions. Published by BMJ.

To cite: Segal JP, Moss AC. Frontline Gastroenterology 2021;12:316-321.

\begin{abstract}
The initial phases of the global SARS-CoV2 pandemic had significant implications for the management of patients with inflammatory bowel disease (IBD). This impact is likely to be sustained and far-reaching across all models of care. Initial questions about the risk of SARSCoV2 infection, and COVID-19 complications, in patients taking maintenance anti-TNFs, JAK inhibitors and other immune modulators have preliminary data. Current models for SARS-CoV-2 transmission predict intermittent outbreaks until 2022, which could disrupt clinical care and negatively affect outcomes for many patients across the globe. This review summarises changes in IBD clinical practice that will be required during the 'post-peak' phase of viral pandemics.
\end{abstract}

\section{COVID-19 IN PATIENTS WITH IBD DURING INITIAL OUTBREAK}

COVID-19 rapidly expanded from a region with a low prevalence of inflammatory bowel disease (IBD) (Wuhan, China) to regions with a high prevalence (eg, New York, USA) in a 3-month timespan. The IBD community had many questions about risks of SAR-CoV-2 infection, and whether special precautions were required in patients taking immunosuppressive therapies. In response, an international registry to capture data on patients with IBD who acquired SARS-CoV2 was established; SECURE-IBD. Over 1000 SARS-CoV2-positive patients with IBD were reported to the IBD SECURE registry as of June $2020 .{ }^{1}$ To date, $32 \%$ have been hospitalised, $6 \%$ required ventilation in the intensive treatment unit and $4 \%$ have died. To put this into context, the reported mortality of COVID-19 has ranged from $6 \%$ (95\% CI 5.4 to 5.8 ) in China to $15 \%$ (95\% CI 12.5 to 17.9 ) outside China. ${ }^{2}$ Global case series reported to date have provided further insights into the impact of COVID-19 in patients with IBD. The first case series from Italy of 522 patients with IBD reported no cases of COVID-19 and no hospitalisations. ${ }^{3}$ Similarly, data from a single-centre database in Spain reported an incidence of COVID-19 of 6.3 per 1000 patients with IBD and concluded that patients with IBD did not have an increased mortality when compared with the general population. ${ }^{4}$

Beyond overall risk of COVID-19, the next question that arose was risk factors for COVID-19 within the IBD population. The first report from the SECURE registry (525 cases of confirmed COVID19) noted that the risk factors for severe COVID-19 were increasing age (adjusted OR (aOR) $1.04,95 \% \mathrm{CI} 1.01$ to 1.02 ), $\geq 2$ comorbidities (aOR 2.9, 95\% CI 1.1 to 7.8), systemic corticosteroids (aOR 6.9, 95\% CI 2.3 to 20.5 ), and sulfasalazine or 5-ASA use (aOR 3.1, 95\% CI 1.3 to 7.7). It is an unexpected finding that 5-ASA are associated with increased severity of COVID-19, as these are not considered to have immunosuppressive qualities. This finding could possibly reflect their widespread use, their utilisation in different healthcare settings and confounders that have yet to be determined. Importantly, TNF antagonist treatment was not associated with severe COVID-19 (aOR 0.9, 95\% CI 0.4 to 2.2). ${ }^{5}$ An Italian cohort described outcomes in 70 patients with COVID-19; 36 patients developed COVID-19-related pneumonia (46\%), 22 (28\%) were hospitalised, 7 (9\%) required non-mechanical ventilation, 9 (11\%) required continuous positive airway pressure therapy, $2(3 \%)$ had endotracheal intubation and $6(8 \%)$ died. Furthermore, four patients $(6 \%)$ were diagnosed with COVID-19 while they were being hospitalised for a severe flare of IBD. ${ }^{6}$ Despite small numbers they reported, that age over 65 years $(p=0.002)$, active IBD $(p=0.02)$ and higher Charlson Comorbidity score were significantly associated with COVID-19-related death; however, 
Table 1 Practical changes to practice

\begin{tabular}{ll}
\hline IBD practice item & Post-Pandemic options \\
\hline Urgent clinic visits & $\begin{array}{l}\text { Pre-visit SARS-CoV-2 testing OR precautions } \\
\text { (masks, gloves) }\end{array}$ \\
Urgent endoscopy & $\begin{array}{l}\text { Pre-procedure SARS-CoV-2 testing OR PPE } \\
\text { precautions }\end{array}$ \\
Patient calls & $\begin{array}{l}\text { Telemedicine, expanded scope for RNs, clinical } \\
\text { pharmacists }\end{array}$ \\
Routine clinic visits & $\begin{array}{l}\text { Telemedicine, at-home calprotectin, remote } \\
\text { monitoring via apps }\end{array}$ \\
\hline
\end{tabular}

PPE, personal protective equipment.

they found no association with poor outcomes related to IBD medications.

Cumulatively, these data have provided reassurance to date for patients with IBD and their clinicians during the lock-down and shielding stage. The next phase will require a more nuanced and logistical challenge for IBD teams to adapt to the 'new normal'.

\section{PLANNING FOR THE NEXT PHASE OF SARS-COV-2}

SARS-CoV-2 is a betacoronavirus, a family of coronaviruses associated with the SARS and MERS syndromes, but also a cause of the 'common cold' (HCoV-OC43 and HCoV-HKU1). Based on prior experience with coronavirus outbreaks, there are two scenarios for the next phase; either a brief intense pandemic followed by eradication (like SARS-CoV-1) or repeated seasonal outbreaks (like HCoV-OC43). ${ }^{7}$ Since both individual (duration of immunity, cross-immunity) and regional factors (control measures) influence these models, IBD teams should plan for assumptions that repeated seasonal outbreaks are likely in the next 2 years. During these outbreaks, logistical restrictions in routine endoscopy, in-person testing/clinic visits and infusion appointments can be expected. Risk mitigation and strategic planning will be required via restructuring of IBD services, as has been necessary during the initial phases of the pandemic (table 1, figure 1).

\section{Maintenance IBD medications during SARS-CoV-2 outbreaks}

As patients with IBD return to clinic, and new patients require initiation of biologic or immunomodulator therapies, we expect on-going inclusion of COVID-19 risk into discussion of therapy options. There are limited data at present on the risk of coronaviruses in patients with IBD. In general, the rates of all viral infections in patients with IBD ranges from $0.1 / 1000$ patient years (no therapy) to $1 / 1000$ patient years (on thiopurine therapy). ${ }^{8}$ The accumulated global cases of COVID-19 among the IBD population $(\sim 1000$ cases with IBD reported among $>2$ million COVID-19 cases worldwide) have been similar across all medication exposure groups. The consensus to date is that medications used to treat IBD do not confer a greater risk of COVID-19 or adverse outcomes from COVID19. In published case series, only high-dose steroid use has been consistently reported as a risk factor for COVID-19 severity and mortality. ${ }^{9} 10$ This is consistent with observations during other coronavirus outbreaks (SARS, MERS) that delayed viral clearance occurred in patients receiving high doses of corticosteroids.

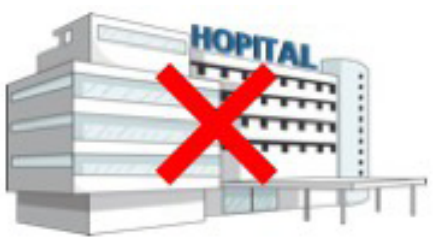

Less Hospital visits
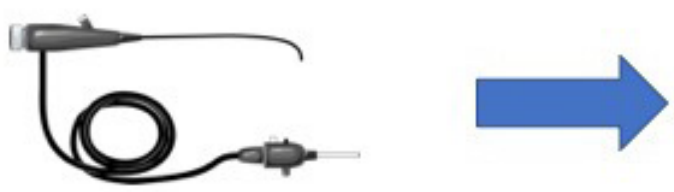

Pre-testing of covid-19 prior to endoscopy

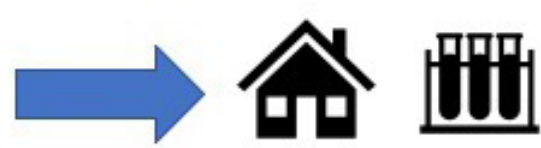

More Home testing
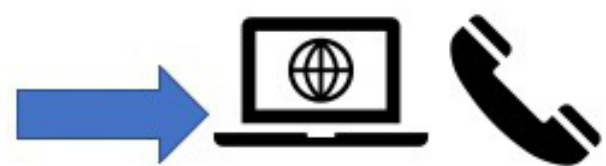

More teleclinics/virtual medicine
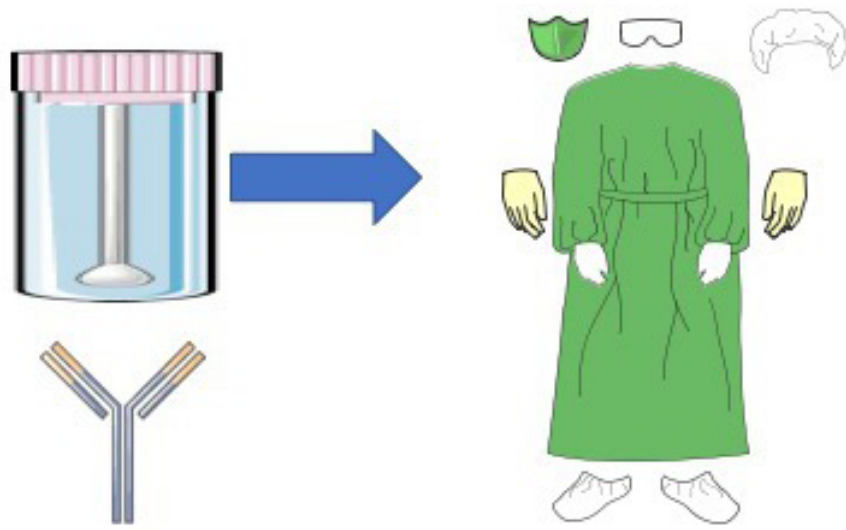

Full Personal Protective Equipment

Figure 1 Graphical representation of IBD practice changes. 
The impact of medications that impair T-cell function (azathioprine, methotrexate, tofacitinib) on coronavirus infection risk or COVID-19 risk will require further data. During the influenza (H1N1) outbreak in 2009, the percentage of affected patients who used thiopurine was comparable with controls, although with a different virus. ${ }^{11}$ Across all classes of drugs for IBD (anti-TNFs, anti-integrins, anti-IL12/23), there are no data from either prior coronavirus outbreaks (SARS, MERS) that a greater risk of infection or complications is associated with these drugs and early experience from the SECURE database supports these findings. ${ }^{10}$ In contrast, there are animal/cell line data that blockade of some cytokine pathways (IL-6, TNF, Th17) by these drug classes could have protective effects against the lung injury seen in COVID-19. ${ }^{12}$ On current evidence, it is advisable that patients with IBD continue their current IBD medications during COVID-19 outbreaks, and if suspected or confirmed COVID-19, discuss their individual situation with their IBD team. This guidance is consistent with International Organization for the Study of Inflammatory Bowel Disease (IOIBD), American Gastroenterological Association (AGA) and British Society of Gastroenterology (BSG) recommendations. ${ }^{13}{ }^{14}$ Based on the reassuring data on medication safety to date, and as additional data become available, patients with IBD will need updated guidance on the need for on-going 'shielding'. During the initial outbreak, the BSG produced guidance on 'shielding' of high-risk groups; those with significant comorbidities and are on an immunosuppressant or are on high-dose steroids, recent dual immunosuppressants, current moderate-severe active disease and those requiring parental support. ${ }^{14}$ In the absence of new data indicating a specific risk of IBD therapies, it appears reasonable to advise these groups to adhere to standard social distancing precautions, rather than more restrictive behaviours than the general population. 'Shielding' may not be sustainable for working adults if the post-pandemic phase includes a prolonged slow decline.

\section{Clinic visits and remote monitoring}

In order to mitigate exposure/infection of patients and staff, the rapid shift that took place to telemedicine and remote monitoring will need to continue during the current pandemic, and future outbreaks. Remote telephone or video consultations will replace in-person clinics for routine follow-up for the foreseeable future. ${ }^{15} 16$ Already, acceptance of this mode of follow-up by physicians had been high; telephone visits or video consultations become the dominant format for clinical assessment in many countries during the peak of the pandemic. ${ }^{17}$ Telemedicine has already proven to be popular with patients, with high adherence and satisfaction scores, by reducing barriers to attendance. ${ }^{18}$
For those patients who require in-person evaluation, clinics will be faced with a choice of either universal pre-visit SARS-CoV-2 testing or continued personal protective equipment (PPE) use for all close-contact interactions. Point-of-care (POC) testing should be considered for IBD clinics; they can rapidly detect SARS-CoV-2 proteins in nasal swab specimens in 5-15 min (eg, Sofia2, Quidel, ID NOW, Abbott). Reported sensitivity for POC antigen or RNA tests ranges from $70 \%$ to $86 \%$, and specificity of $95 \%$, and some have been approved by FDA and CE already. ${ }^{19}$ Most centres already have on-site laboratory PCR testing for SARS-CoV-2 sequences, but the time to results (4-24hours) may make them a less practical solution than POC assays. Once validated serological tests for immunity are available, these should also become part of the routine evaluation for patients with IBD. These procedures will apply to infusion areas as well as clinic spaces.

In addition to person-person interactions, the COVID-19 pandemic has renewed interest in group communication, and remote monitoring of symptoms, biomarkers and biosensors. Since many regions will experience a second wave of the pandemic, the ability to detect deteriorations in disease, and react remotely, will be important. ${ }^{20}$ Many IBD apps are available for smartphones (eg, myIBDcoach) but were used by less than $15 \%$ of clinicians prior to COVID-19. ${ }^{17}$ Like televisits, the regulatory hesitation around using these monitoring methods have relaxed in recent months, and asking patients to record and share symptom diaries via this medium should be enlisted to screen for disease flare-ups. Evidence from at least one randomised controlled trial has reported a reduction in hospitalisations and outpatient visits in patients using such a program. ${ }^{21}$ Similarly, faecal calprotectin (FC), an established disease and prognosis marker, should be incorporated into remote monitoring, ideally using home kits for sample acquisition. As noted later on, POC assays for FC can provide objective evaluation in symptomatic patients, minimising the need for endoscopic procedures and blood tests.

Patient education and advice will remain important during all phases of COVID-19 pandemics, and IBD centres should develop mechanisms to quickly disseminate critical advice to their patients. The IBD centre in Wuhan, China, provided advice and education through social media to their patients with IBD based on a database of all patient contacts once the outbreak began. ${ }^{22}$ One of the author's program (AM) used a direct messaging service to text message all patients with IBD to continue their biologics when the regional lock-down began. These patient databases will allow teams to provide urgent updates and advice in order to help protect patients with IBD from future waves of COVID-19.23 


\section{Endoscopy}

During the decline of the pandemic, and subsequent outbreaks, a strategy for stratifying patients for elective endoscopy for IBD will be required. ${ }^{24}$ All IBD surveillance and staging that was deferred during the initial pandemic will need staged resumption on a stratified basis. In the post-pandemic phase, centres should prioritise the highest risk groups for resumption of delayed surveillance; those with a history of dysplasia or primary sclerosing cholangitis. Similarly, faecal calprotectin levels should replace endoscopy as a means to confirm mucosal healing or assess symptom relapse in most cases.

While community prevalence of COVID-19 persists, re-establishment of elective endoscopy services will rely on the availability of local testing for SARS-CoV-2 prior to procedures, per BSG guidance. ${ }^{25}$ If SARS-CoV-2 status is unknown, full PPE should be used, negative pressure rooms are preferred, and full decontamination of the room and equipment should follow. $^{26}$ Endoscopy units should review POC tests for SARS-CoV-2 status and identify an option with simple workflow, and rapid test results as part of the patient admission process. When community prevalence declines, a background threshold could be determined where standard endoscopy precautions can be safely used again in SARS-CoV-2-negative patients. A report from an intermediate prevalence region $(12 \%$ prevalence, Miami-Dade county, USA) described only 1 SARS-CoV-2-positive person from 396 screened before endoscopy $(0.25 \%){ }^{27} \mathrm{~A}$ unique factor related to colonoscopy is the potential for viral particles to be present in the stool for several weeks after recovery. ${ }^{28}$ It remains unclear if there is active virus and a real transmission risk ${ }^{29}$ and in a single centre it has been demonstrated that the risk of transmission from COVID-19 during endoscopy is very low. ${ }^{30}$ Despite these findings, current guidelines suggest that caution is taken in performing colonoscopy in patients after COVID-19.

The management of IBD flare-ups during the SARS-COV2 pandemic has its own specific challenges. The challenges come from protecting patients with IBD from hospitalisation where possible, selecting appropriate IBD therapies while mitigating the risk of COVID-19 complications, and ensuring timely investigations and treatment. The first challenge is in distinguishing IBD flare-ups from COVID-19 infection. There is significant overlap between COVID-19 symptoms and IBD, with GI symptoms reported in up to $79 \%$ of COVID-19 cases. In a meta-analysis, it was found that $18 \%$ of patients with SARS-COV2-19 had gastrointestinal (GI) symptoms and $48 \%$ of patients had faecal SARS-COV2 RNA detected even after cessation of respiratory symptoms. ${ }^{31}$ Despite this, other case series have highlighted that GI disturbances secondary to COVID-19 are present in only $2 \%$ of cases and that rectal bleeding is $\operatorname{rare}^{2632}$; in view of this, a typical history suggestive of IBD with biochemical evidence in the absence of symptoms of signs of COVID-19 is likely to represent IBD.

To aid diagnosis in view of potential overlap in symptoms and biochemistry, further rapid and accurate tests for SARS-CoV-2 infection (PCR/immunoassay) and immunity (ELISA) will prove useful in the post-pandemic phase. Furthermore, the impact of COVID-19 on faecal calprotectin assays will need to be further elucidated to validate this test in the COVID-19 era. Clinical centres should consider incorporating stool assays for faecal SARS-CoV-2, alongside faecal calprotectin, as an important tool to distinguish IBD flare-ups from COVID-19. It has also been documented that COVID-19 causes elevations in inflammatory markers such as $\mathrm{CRP}^{33}$ and faecal calprotectin. ${ }^{34}$

In one small series, it was reported that COVID-19 can induce an inflammatory response in the bowel even in asymptomatic patients. ${ }^{34}$ It is currently poorly understood the implications this has in differentiating IBD from COVID-19. However, in the context of symptoms suggestive of IBD we believe that faecal calprotectin can still be a valuable test to distinguish IBD from other disorders.

The second challenge will be therapy selection when SARS-CoV-2 status is unknown, or during times of high community prevalence. As noted previously, apart from high-dose steroids, none of the currently used agents for IBD confer an increased risk of COVID-19 complications. Thus, the most suitable agent for each individual case should be considered, given prior medication exposure and availability. In view of the potential risk of steroids, patients with IBD experiencing a flare will need access to rapid SARS-CoV2 testing to help balance benefits of disease control against risks of steroids during COVID-19 infections. Importantly, steroids have also been shown to have a negative effect on COVID-19 outside of IBD to include increase risk of osteonecrosis, ${ }^{35}$ higher mortality, longer length of stay and higher rates of bacterial infections. ${ }^{36}$ It is therefore important that steroid alternatives are considered where possible and careful risk assessment undertaken for each patient.

When a patient with IBD has acquired COVID19 , the advice is to where possible stop any immunosuppressive medications such as thiopurines and biologics, on a case-by-case basis. Steroids should not be suddenly stopped but patients should discuss any ongoing steroid use with their team.

\section{Summary of BSG, ECCO, AGA and IOIBD guidance}

Several international expert groups have presented interim guidance on management of patients with IBD during current phase (widespread human infection) of the COVID-19 pandemic (table 2). These represent 'best practice' advice until more evidence-based recommendations can be developed. Since the quantification of population immunity, and accuracy of diagnostic 
Table 2 Societal guidance during the COVID-19 pandemic

\begin{tabular}{ll}
\hline Organisation & Link \\
\hline IOIBD & $\begin{array}{l}\text { https://www.gastrojournal.org/article/S0016- } \\
\text { 5085(20)30465-0/fulltext }\end{array}$ \\
BSG & https://www.bsg.org.uk/covid-19-advice \\
AGA & https://www.gastrojournal.org/article/S0016- \\
& 5085(20)30482-0/pdf \\
ECCO & https://ecco-ibd.eu/publications/covid-19.html \\
\hline
\end{tabular}

testing, remains in evolution, these recommendations may need to be adapted locally as prevalence, testing and PPE access fluctuate.

Our knowledge regarding SARS-CoV-2 and its effect on patients with IBD remains in its infancy. Sufficient cases have been accumulated in the SECURE-IBD registry, with comparisons across medication exposure and disease activity groups, to determine that there is no increased risk from maintenance medications used during clinical remission. The safety of starting steroids, anti-TNFs and other immunosuppressants in patients with active IBD and in patients who are infected with SARS-CoV-2 will be another important data point for case-by-case management decisions. At a micro level, since live SARS-CoV-2 can be detected in faecal samples, the risk of faecal transmission to household contacts and medical staff needs to be investigated. At a macro level, the focus is on how restructuring of IBD services may impact on patients with IBD to help teams address any unmet needs. Missed infusions, blood monitoring and surveillance colonoscopies all have potential negative impacts on outcomes for our patients with IBD. Once a SARS-CoV-2 vaccine is available, its immunogenicity in patients with IBD will still need to be determined. The 2009 vaccine to $\mathrm{H} 1 \mathrm{~N} 1$ produced a low rate of seroprotection in patients with IBD (50\%), particularly among those who were immunosuppressed. ${ }^{37}$

Contributors JPS and ACM wrote the manuscript and contributed equally. Both approved final version.

Funding The authors have not declared a specific grant for this research from any funding agency in the public, commercial or not-for-profit sectors.

Competing interests Both are on the editorial board on Frontline Gastroenterology.

Patient consent for publication Not required.

Provenance and peer review Not commissioned; externally peer reviewed.

This article is made freely available for use in accordance with BMJ's website terms and conditions for the duration of the covid-19 pandemic or until otherwise determined by BMJ. You may use, download and print the article for any lawful, non-commercial purpose (including text and data mining) provided that all copyright notices and trade marks are retained.

\section{ORCID iDs}

Jonathan P Segal http://orcid.org/0000-0002-9668-0316 Alan C Moss http://orcid.org/0000-0003-0179-5477

\section{REFERENCES}

1 Current data, SECURE-IBD database. Available: https:// covidibd.org/current-data/ [Accessed 13 Apr 2020].

2 Baud D, Qi X, Nielsen-Saines K, et al. Real estimates of mortality following COVID-19 infection. Lancet Infect Dis 2020;0. doi:10.1016/S1473-3099(20)30195-X

3 Norsa L. Uneventful course in IBD patients during SARSCoV-2 outbreak in northern Italy. Gastroenterology 2020.

4 Taxonera C, Sagastagoitia I, Alba C, et al. 2019 novel coronavirus disease (COVID-19) in patients with inflammatory bowel diseases. Aliment Pharmacol Ther 2020;382.

5 Brenner EJ, Ungaro RC, Gearry RB, et al. Corticosteroids, but not TNF antagonists, are associated with adverse COVID-19 outcomes in patients with inflammatory bowel diseases: results from an international registry. Gastroenterology 2020.

6 Bezzio C, Saibeni S, Variola A, et al. Outcomes of COVID-19 in 79 patients with IBD in Italy: an IG-IBD study. Gut 2020;69:1213-7.

7 Kissler SM, Tedijanto C, Goldstein E, et al. Projecting the transmission dynamics of SARS-CoV-2 through the postpandemic period. Science 2020;368:860-8.

8 Kirchgesner J, Lemaitre M, Carrat F, et al. Risk of serious and opportunistic infections associated with treatment of inflammatory bowel diseases. Gastroenterology 2018;155:33746.

9 Li X, Xu S, Yu M, et al. Risk factors for severity and mortality in adult COVID-19 inpatients in Wuhan. J Allergy Clin Immunol 2020. doi:10.1016/j.jaci.2020.04.006

10 Current summary data, SECURE-IBD database. Available: https://covidibd.org/current-data/ [Accessed 19th Apr 2020].

11 Naganuma M, Fujii T, Kunisaki R, et al. Incidence and characteristics of the 2009 influenza (H1N1) infections in inflammatory bowel disease patients. J Crohns Colitis 2013;7:308-13.

12 Neurath MF. COVID-19 and immunomodulation in IBD. Gut 2020;69:1335-42.

13 Rubin DT, Feuerstein JD, Wang AY, et al. AGA clinical practice update on management of inflammatory bowel disease during the COVID-19 pandemic: expert commentary. Gastroenterology 2020. doi:10.1053/j.gastro.2020.04.012

14 Kennedy NA, Jones G-R, Lamb CA, et al. British Society of Gastroenterology guidance for management of inflammatory bowel disease during the COVID-19 pandemic. Gut 2020;69:984-90.

15 Danese S, Cecconi M, Spinelli A. Management of IBD during the COVID-19 outbreak: resetting clinical priorities. Nat Rev Gastroenterol Hepatol 2020;17:253-5.

16 Fiorino G, Allocca M, Furfaro F, et al. Inflammatory bowel disease care in the COVID-19 pandemic era: the Humanitas, Milan, experience. J Crohn's Colitis 2020;24.

17 Lees CW, Regueiro M, Mahadevan U. Innovation in IBD care during the COVID-19 pandemic: results of a global telemedicine survey by the International Organization for the Study of Inflammatory Bowel Disease. Gastroenterology 2020.

18 Cross RK, Arora M, Finkelstein J. Acceptance of telemanagement is high in patients with inflammatory bowel disease. J Clin Gastroenterol 2006;40:200-8.

19 Castro R, Luz PM, Wakimoto MD, et al. COVID-19: a meta-analysis of diagnostic test accuracy of commercial assays registered in Brazil. Braz J Infect Dis 2020;24:180-7.

$20 \mathrm{Xu} \mathrm{S,} \mathrm{Li} \mathrm{Y.} \mathrm{Beware} \mathrm{of} \mathrm{the} \mathrm{second} \mathrm{wave} \mathrm{of} \mathrm{COVID-19.} \mathrm{Lancet}$ 2020;395:1321-2.

21 de Jong MJ, van der Meulen-de Jong AE, Romberg-Camps MJ, et al. Telemedicine for management of inflammatory bowel disease (myIBDcoach): a pragmatic, multicentre, randomised controlled trial. Lancet 2017;390:959-68.

22 An P, Ji M, Ren H, et al. Prevention of COVID-19 in patients with inflammatory bowel disease in Wuhan, China. Lancet Gastroenterol Hepatol 2020;5:525-7. 
23 Segal JP, Quraishi MN, Bhala N, et al. Prevention of COVID-19 in patients with IBD. Lancet Gastroenterol Hepatol 2020;640. doi:10.1016/S2468-1253(20)30153-9

24 The British Society of Gastroenterology. COVID-19 Guidance \& Advice. Available: https://www.bsg.org.uk/covid-19-advice/ [Accessed 13 Apr 2020].

25 The British Society of Gastroenterology. Endoscopy activity and COVID-19: BSG and JAG guidance. Available: https:// www.bsg.org.uk/covid-19-advice/endoscopy-activity-andcovid-19-bsg-and-jag-guidance/?utm_source $=$ Members\& $\mathrm{utm}$ _campaign $=$ ffd002b62b-EMAIL_CAMPAIGN_2020_02_ 13_03_21_COPY_01\&utm_medium $=$ email\&utm_term $=0$ _ be5fefa54d-ffd002b62b-\&mc_cid $=$ ffd002b62b\&mc_eid $=\%$ 5B [Accessed 4 May 2020].

26 Iacucci M, Cannatelli R, Labarile N, et al. Endoscopy in inflammatory bowel diseases during the COVID-19 pandemic and post-pandemic period. Lancet Gastroenterol Hepatol 2020;5:598-606.

27 Forde JJ, Goldberg D, Sussman D, et al. Yield and implications of pre-procedural COVID-19 PCR testing on routine endoscopic practice. Gastroenterology 2020. doi:10.1053/j. gastro.2020.05.062

28 Wu Y, Guo C, Tang L, et al. Prolonged presence of SARSCoV-2 viral RNA in faecal samples. Lancet Gastroenterol Hepatol 2020;5:434-5.
29 Wölfel R, Corman VM, Guggemos W, et al. Virological assessment of hospitalized patients with COVID-2019. Nature 2020;581:1-5.

30 Repici A, Aragona G, Cengia G, et al. Low risk of COVID-19 transmission in Gi endoscopy. Gut 2020;69:1925-7.

31 Cheung KS. Gastrointestinal manifestations of SARS-CoV-2 infection and virus load in fecal samples from the Hong Kong cohort and systematic review and meta-analysis. Gastroenterology 2020.

32 Wang D, Hu B, Hu C, et al. Clinical characteristics of 138 hospitalized patients with 2019 novel coronavirus-infected pneumonia in Wuhan, China. JAMA 2020;323:1061.

33 Lescure F-X, Bouadma L, Nguyen D, et al. Clinical and virological data of the first cases of COVID-19 in Europe: a case series. Lancet Infect Dis 2020;20:697-706.

34 Effenberger M, Grabherr F, Mayr L, et al. Faecal calprotectin indicates intestinal inflammation in COVID-19. Gut 2020;69:1543-4.

35 Tang C, Wang Y, Lv H, et al. Caution against corticosteroidbased COVID-19 treatment. Lancet 2020;395:1759-60.

36 Yang Z, Liu J, Zhou Y, et al. The effect of corticosteroid treatment on patients with coronavirus infection: a systematic review and meta-analysis. J Infect 2020;81:59.

37 Cullen G, Bader C, Korzenik JR, et al. Serological response to the $2009 \mathrm{H} 1 \mathrm{~N} 1$ influenza vaccination in patients with inflammatory bowel disease. Gut 2012;61:385-91. 